\title{
Prospective multicenter comparison of proliferation and other prognostic factors in lymph node negative lobular invasive breast cancer
}

\author{
Einar Gudlaugsson - Ivar Skaland · Emiel A. M. Janssen · Paul J. van Diest • \\ Feja J. Voorhorst $\cdot$ Kjell Kjellevold · Axel zur Hausen · Jan P. A. Baak
}

Received: 30 December 2008/Accepted: 9 June 2009/Published online: 1 July 2009

(C) Springer Science+Business Media, LLC. 2009

\begin{abstract}
Evaluation of prognostic factors in lymph node negative (LNneg) invasive lobular cancers (ILCs). Prospective analysis of proliferation and other prognosticators in 121 LNneg ILCs (119 months median follow-up, range 19-181), without adjuvant chemotherapy. ILC subtype was assessed in accordance with WHO-2003 criteria. Immunohistochemical E-cadherin and estrogen receptor were used. With a median follow up time of 83 months (range 19-181), 30 of the 121 (25\%) ILC patients developed distant metastases and 27 (22\%) died. None of the cases classified as solid/pleomorphic lobular were E-cadherin or estrogen receptor positive, contrasting the other ILCs. The solid/alveolar ILCs $(n=17)$ had a worse survival $(50 \%)$ than the other ILCs $(n=104 ; 83 \%, P<0.0001)$. Mitotic activity index (MAI) (but not nuclear grade or tubule
\end{abstract}

E. Gudlaugsson - I. Skaland · E. A. M. Janssen ·

K. Kjellevold · J. P. A. Baak

Department of Pathology, Stavanger University Hospital,

Box 8100, 4068 Stavanger, Norway

E. Gudlaugsson · I. Skaland · J. P. A. Baak ( $\bowtie)$

The Gade Institute, University of Bergen, Bergen, Norway

e-mail: jpabaak@yahoo.com

J. P. A. Baak

Free University, Amsterdam, The Netherlands

P. J. van Diest

Department of Pathology, University Medical Center,

Utrecht, The Netherlands

\section{F. J. Voorhorst}

Department of Epidemiology and Biostatistics, VU University

Medical Center, Amsterdam, The Netherlands

A. z. Hausen

Department of Pathology, University Hospital Freiburg,

Freiburg, Germany formation) was prognostic with a threshold $0-5$ versus $>5$ (=MAI-5) (contrasting MAI $<10$ vs. $\geq 10$ in breast cancers in general; 85 and $54 \%$ survival, $P<0.0001$ ). In multivariate analysis only subtype and MAI but none of the other characteristics had independent prognostic value. Histologic subtype and MAI have independent prognostic value in node negative invasive lobular cancers.

Keywords Lobular invasive breast cancer - Prognosis . Proliferation - Mitotic activity index

\section{Introduction}

Invasive lobular cancers (ILCs) form about $10 \%$ of all primary breast carcinomas, and the incidence may be increasing [1]. It is generally believed that ILC is a different entity from invasive ductal carcinoma (IDC) as the response to pre-operative chemotherapy in ILCs may be worse, with a greater need for rescue mastectomy. This lesser response in ILC than in IDC is correlated to their immunohistochemical profile [2]. It is thus important that the biological prognostic characteristics of ILCs should be studied further in order to make the fine-tuning of treatment possible [3].

A classical study [4] analyzed the factors which are prognostic in ILC. In addition to the disease stage at presentation, the other major significant factor in predicting survival was histological subtype. A recent very large study compared 4,140 ILC and 45,169 (not otherwise specified) IDC patients [5] and found that despite the fact that the biological phenotype of ILC seemed quite favorable, the clinical outcome of those patients was not better than that of patients with IDC. The authors concluded that different management decisions should be based on individual 
patient and tumor biologic characteristics, than lobular histology.

Immunohistochemical analysis of E-cadherin has led to the identification of new variants of lobular carcinoma associated with a more aggressive clinical behavior [6]. Proliferation variables such as tritiated thymidine labeling index and mitotic activity index (MAI) are strong prognostic factors in invasive breast cancer [7]. The MAI is also prognostic in small tumors of $<1 \mathrm{~cm}$, and grades $1+2$ tumors between 1 and $3 \mathrm{~cm}$ diameter, which are not usually treated with adjuvant chemotherapy [8]. It was found that the histologic grade (in which the MAI plays an important role) is prognostic in ILC, but the different constituents of grade (MAI, tubule formation, nuclear atypia) were not analyzed separately [8]. Proliferation is usually low in ILCs, though in rare cases mitoses can be frequent. As it is uncertain what the prognostic value of this phenomenon is in ILCs, we have prospectively studied these interesting occurrences in comparison with other characteristics in the large multicenter material of the MMMCP study [7], involving 2,274 invasive breast cancers.

\section{Patients and methods}

\section{Patients}

Details of the prospective multicenter MMMCP study have been described elsewhere [7]. The study has been approved by the Regional Ethics Committee of the VU Medical center and collaborating hospitals. All consecutive primary invasive breast cancer patients diagnosed in the collaborating hospitals were enrolled, and the follow-up was updated annually. The guidelines for reporting tumor marker studies [9] were followed. Out of 3,479 MMMCP patients registered, 138 were not invasive; 10 had sarcomas; 32 had metastases at the time of diagnosis; 235 were not operable or the operation was not curative from the onset; 242 had previous or concurrent malignancies (other than basal cell carcinoma or cervical carcinoma in situ $=$ CIS); 42 were double sided, and 460 were lost to follow-up. This left 2,274 patients with invasive cancers for further study. In 92 patients including 4 invasive lobular cancers, no axillary lymph node dissection was done for various reasons (old age $n=46$, already distant metastases at the time of operation $n=6$, patient refused axillary dissection $n=6$, cancer diameter $>5 \mathrm{~cm} n=5$, thoracic wall invasion $n=7$, poor clinical non-breast cancer-related conditions $n=12$, reason unknown $n=10$ ). All remaining 2,182 patients were treated with modified radical mastectomy (MRM, $n=1,141$ ) or breast-conserving therapy (BCT, $n=1,041$ ), always with adequate axillary lymph node dissection (median 11 lymph nodes detected in the axillary lymph node dissection specimens). Figure 1 illustrates which patients from the prospective multicenter MMMCP study were used in the current study on lymph node negative lobular invasive breast cancer. Loco-regional radiotherapy was given in cases that underwent breastconserving therapy or had medially localized tumors. None of the patients received any form of adjuvant chemo or hormonal therapy.

\section{Pathology}

Post-surgical size of the tumor (pT) was measured in the fresh specimens; the tumors were cut into slices of $0.5 \mathrm{~cm}$, fixed in buffered $4 \%$ formaldehyde, and embedded in paraffin. Paraffin sections of $4 \mathrm{~mm}$ thickness were cut and stained with hematoxylin and eosin. Histologic grade and type were assessed in many participating centers and independently reviewed by three of us (JB, PD and E.G) with considerable experience in breast pathology. Invasive lobular cancers were defined using the World Health Organization (WHO) criteria [1] as cancers composed of non-cohesive cells individually dispersed or arranged in single-file linear pattern (often referred to as 'Indian filing'), often associated with lobular carcinoma in situ $=$ LCIS, frequently with a diffuse growth pattern or cell infiltrate, and usually with a concentric "targetoid" pattern around normal ducts. Grade was assessed, again according to the WHO criteria using MAI $0-5=1,6-10=2$ and $>10$ as 3 , nuclear atypia as mild $=1$, moderate $=2$ and marked $=3$, and tubule formation as much $(>75 \%)=1$, minimal $(<10 \%)=3$ and intermediate $(10-75 \%)=2$. At careful review of type 1,812 cancers were ductal; 113 ductal combined with other types (but non-lobular); 201 lobular invasive; 23 tubular; 22 colloid; 11 medullary, and 2 were papillary (total $=2,184$ ). As the non-lobular nonductal cancers were relatively rare and also had a much better prognosis than the ILCs and IDCs, they were not further considered in the present study.

Of the 201 lobular invasive cancers, 121 were LNneg, and sub-typed independently by two of us (E.G, JB). LCIS was characterized by extended lobules with a solid typical cell pattern without lumina, consisting of cells with remarkably round and regular nuclei with regular spacing, often with somewhat more cytoplasm than in ductal cancers and frequently containing cytoplasmic vacuoles. The pleomorphic and solid variants may cause differential diagnostic problems with classical ("Indian filing") ILC on one hand (for the pleomorphic ILCs) and ductal grade 3 cancers (for the solid ones) on the other. To avoid this, using WHO 2004 criteria we defined ILCs as pleomorphic if they had clearly large and round rather than molded small nuclei located in Indian files of one, or at maximum, focally two cells thickness. To avoid a false inclusion of 
Fig. 1 Flow-sheet diagram depicting which patients from the prospective multicenter MMMCP study were used for the current study on lymph node negative lobular invasive breast cancer

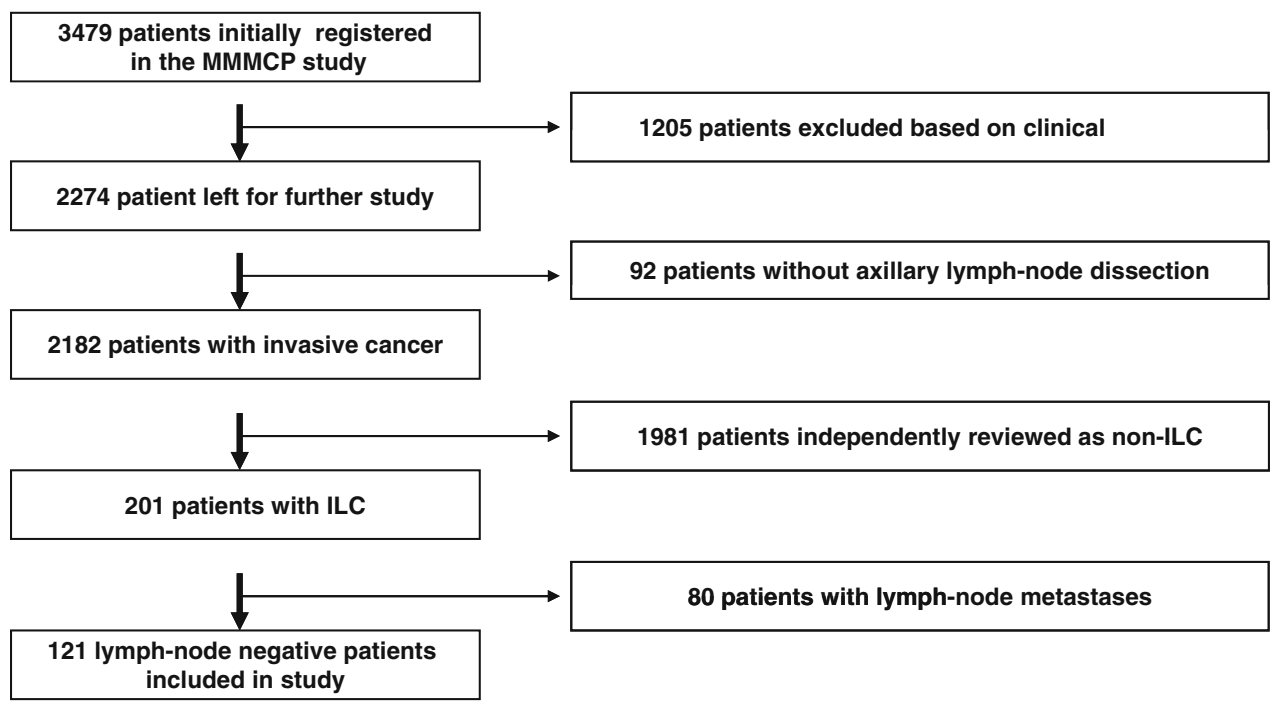

ductal grade 3 cancers, the following characteristics of LCIS or Indian filing with targetoid growth pattern should be present to classify a cancer as an ILC, solid subtype. Presence of extensive necrosis occurred in one solid cancer which is unusual in solid ILCs, and therefore this case was excluded as well, also due to the E-cadherin staining result. In all cases classified as pleomorphic or solid, E-cadherin staining was done using normal glands in the same sections as internal controls. The non-lobular ductal combinations were regarded as ductal, but the ductulo-lobular cancers were regarded as a lobular subtype variant; they had the same survival as the ductal and the non-solid-non-pleomorphic lobular subtype cancers. The resection margins in the biopsies were evaluated as free or not free of tumor. Estrogen receptor value (ER) was assessed in reference laboratories by charcoal technique (cut off $10 \mathrm{fmol} / \mathrm{mg}$ protein). For the ER negative cases, immunohistochemical analysis was done.

\section{Immunohistochemistry}

E-Cadherin was done as described in all solid and pleomorphic cases to exclude E-Cadherin positive grade 3 ductal cancers. Antigen retrieval and antibody dilution were optimized prior to the study onset. To ensure uniform handling of samples, all sections were processed simultaneously. Paraffin sections adjacent to the H\&E sections used for assessment of MAI and histology were dried overnight at $37^{\circ} \mathrm{C}$ followed by $1 \mathrm{~h}$ at $60^{\circ} \mathrm{C}$. Sections were deparaffinized in xylene and rehydrated by decreasing the concentration of alcohol. Antigen was retrieved from a highly stabilized retrieval system (ImmunoPrep, Instrumec, Oslo, Norway) using $10 \mathrm{mM}$ Tris/1 mM EDTA (pH 9.0) as the retrieval buffer. Sections were heated for $3 \mathrm{~min}$ at $110^{\circ} \mathrm{C}$ followed by $10 \mathrm{~min}$ at $95^{\circ} \mathrm{C}$ and cooled to $20^{\circ} \mathrm{C}$.
Rabbit monoclonal ER (clone SP1 Thermo Scientific, Fremont, USA) was used at a dilution of $1 / 400$ and E-Cadherin (Clone 36B5, Novocastra Laboratories ltd, Newcastle upon Tyne, UK) was used at a dilution of 1/50. The sections were incubated for $45 \mathrm{~min}$ at $22^{\circ} \mathrm{C}$. Dako antibody diluent (S0809) was used. Endogenous peroxidase activity was blocked with a peroxidase-blocking reagent (S2001; Dako) for 10 min. The immune complex was visualized by the Dako REAL EnVision Detection System, Peroxidase/DAB, Rabbit/Mouse (K5007; Dako). Sections were incubated with EnVision/HRP, Rabbit/Mouse for $30 \mathrm{~min}$, and diaminobenzidine $(\mathrm{DAB}+)$ chromogen for $10 \mathrm{~min}$. The sections were counterstained with hematoxylin, dehydrated, and mounted. All steps were performed using Dako Autostainer and TBS (S1968; Dako) with $0.05 \%$ Tween 20 as wash buffer.

Mitosis counts and nuclear morphometry

Following the MMMCP protocol, the total number of welldefined mitotic figures was counted at the time of diagnosis at $400 \times$ magnification (objective 40, field diameter $450 \mu \mathrm{m}$ at specimen level) in 10 consecutive neighboring fields of vision in the most poorly differentiated peripheral area of the tumor (=measurement area, representing a total area of $1.59 \mathrm{~mm}^{2}$ ). Fields with necrosis or inflammation were avoided, and doubtful structures were ignored. The resulting total number of mitosis in the ten fields of vision is the MAI. An accurate MAI assessment takes 3-5 min. Correction of MAI for the percentage of tissue occupied by stroma or the number of tumor cells was not applied since it was previously shown that this does not substantially improve the prognostic value of the MAI and ismore time consuming. The MAI is a continuous variable, and according to many previous studies, the most important 
prognostic threshold for the whole group is $\mathrm{MAI}<3$ (excellent prognosis), 3-10 (favorable prognosis) versus $\geq 10$ (poor prognosis). We also analyzed the classical Nottingham thresholds $(0-5,6-10$ and $>10)$.

Nuclear morphometric analysis of the representative H\&E sections used for revision grading was performed with an image analysis system (Leica, Cambridge, UK) at $1,800 \times$ screen magnification, using rigid point-weighted systematic random sampling [10]. This guarantees unbiased high reproducibility and stronger prognostic value [11]. At least 50 and a maximum of 225 (median: 79) nuclei were measured per case (one nucleus per field of vision) and the mean nuclear area was calculated. The total number of nuclei per case was determined by the coefficient of error (CE) of the ongoing measurements per case. The CE was continuously calculated and if it fell under 5\%, the measurement was terminated but only if a minimum of 50 nuclei was measured. Intra- and inter-observer reproducibility of this method has previously proven to be very high [12].

\section{Statistical analysis}

Main endpoints were distant recurrence (Metastases-Free Survival $=$ MFS) and mortality due to distant metastases (Overall Survival = OS). In analyzing the probability that patients would remain free of distant metastases, we defined recurrence as any first recurrence at distant sites. All other patients were censored on the date of the last follow-up visit and included deaths from causes other than breast cancer, local, or regional recurrences or the development of a secondary primary cancer (including contra-lateral breast cancer). Mortality was defined as any death due to distant metastases (as evident from clinical, radiologic, histologic, or autopsy data) (no patients died from loco-regional disease). If the cause of death was unknown, but a metastasis was previously detected, then death was considered as breast cancer related unless explicitly stated otherwise (in line with other studies) [1]. If the status during follow-up indicated a confirmed metastasis without a date of recurrence, the date of that follow-up visit was used. SPSS version 13 (SPSS, Chicago, USA) was used for the analyses. Age, time to first recurrence, and survival time were calculated relative to the date of primary diagnosis. Continuous variables were discretized according to previously described thresholds, or dividing the whole group into subgroups according to the medians, tertiles, or quartiles. Moreover, Receiver Operator Curves were used for assessing the optimal threshold. Survival curves were constructed using Kaplan-Meier techniques. Differences between groups were tested by log-rank tests or tests for trend. The relative importance of potential prognostic variables was tested using Cox-proportional hazard analysis and expressed in Hazards Ratio (HR) with $95 \%$ confidence intervals.

\section{Results}

None of the invasive lobular cancers classified as solid/ pleomorphic lobular were E-Cadherin or estrogen receptor positive, contrasting the other ILCs. With a median followup time of 83 months (range: 19-181), thirty of the 121 (25\%) ILC patients developed distant metastases and of these $27(22 \%)$ died. The histological ILC subtype was a strong prognosticator, but only the survival difference between the Solid and Alveolar (=SA) ILC subtypes together $(n=17)$ versus all other subtypes was significant. Therefore, SA-ILCs had a much worse prognosis than the other ILCs (41 versus $84 \%$ survival, $P<0.0001$, Hazard Ratio $=6.0,95 \%$ Confidence Interval $=95 \% \mathrm{CI}=2.8$ 13.0; Fig. 2). The MAI (but not nuclear grade, nor tubular formation or histologic grade) was prognostic but the strongest threshold was $0-5(n=93)$ versus $>5[n=28$; MAI-5; contrasting the previously described threshold of $0-9$ vs. $\geq 10$ in general (mostly ductal) cancers]; the survival rates of 10 years were 85 and $54 \%, P<0.0001$, $\mathrm{HR}=3.9,95 \% \mathrm{CI}=1.8-8.4$ (Fig. 3). The other features were (less) significant $(0.05<P<0.01)$ with univariate survival analysis.

With multivariate analysis, histologic subtype (as SAILCs versus all other subtypes) and MAI had independent statistical and clinically relevant value (in sequence of decreasing significance; $P<0.0001)$. This combination overshadowed all other univariately significant features. MAI-5 added to the histologic subtype but this was significant only in the non-SA ILCs (MAI 0-5 and >5: 87 and

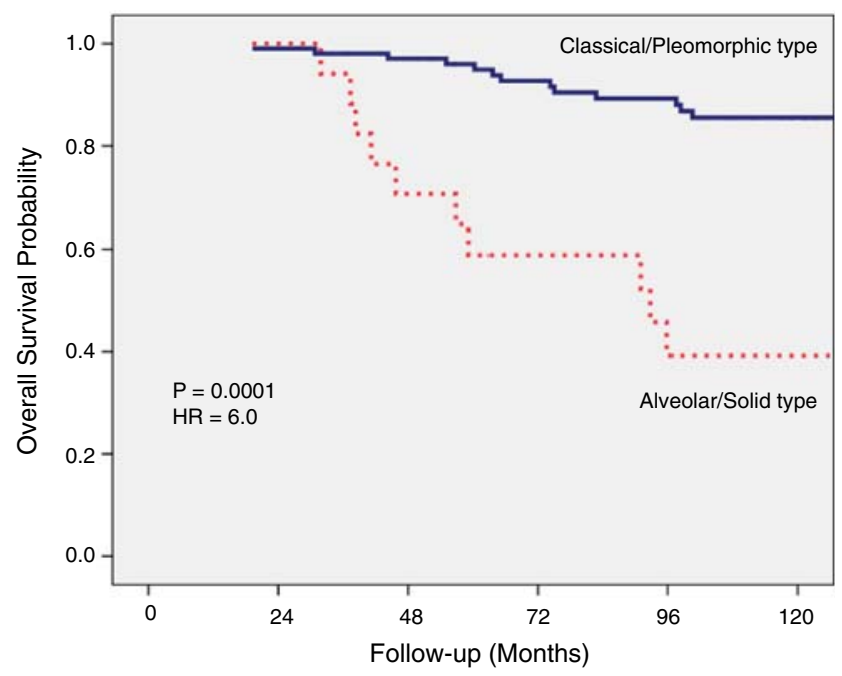

Fig. 2 Survival curves of the lobular invasive cancers stratified over histologic subtype 


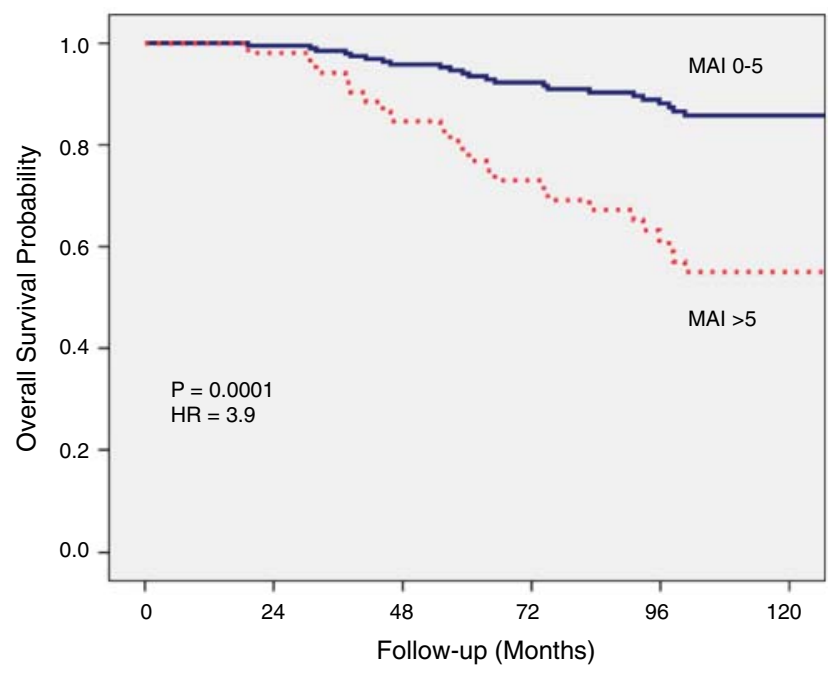

Fig. 3 Survival curves of the lobular invasive cancers stratified over mitotic activity index. Note that the important prognostic threshold in these lobular invasive cancers is 5 . In ductal cancers, the strongest prognostic threshold is 10

$67 \%$ overall survival respectively, $P=0.04, \mathrm{HR}=2.8$, $95 \% \mathrm{CI}=1.1-7.6)$ and not in the SAs-ILC (57 and 30\% overall survival, $P=0.42$ ).

\section{Discussion}

The current large prospective multicenter material on lymph node negative invasive lobular cancers without systemic adjuvant treatment showed that the characteristics in the present study are comparable to other previous studies, making the results representative for LNneg lobular invasive cancer in general. ILC-subtype and MAI are prognostically the most important. The fact that MAI was prognostic, rather than tubule formation, nuclear atypia and grade is in agreement with a previous study on breast cancer in general [13]. What is new is the fact that MAI with a threshold of five and not ten is one of the three most important prognosticators in the lobular invasive cancers, which explains why the prognostic value of the MAI in breast cancer varies in different studies. Using the classical prognostic threshold of ten works well for the ductal but not for the ILC subtype cancers, whereas in the ILC cancers, MAI-5 will prognosticate best. Consequently, depending on the mix of ductal and lobular cancers, the prognostic thresholds of the MAI will vary. The prognostic value of the MAI is therefore distorted when histologically different groups are mixed (i.e., ductal and lobular cancers) with prognostically different MAI thresholds.

The fact that histologic subtype appeared to be a strongly significant prognosticator is in agreement with a previous classical study using both lymph node negative and positive ILCs [14] (we studied lymph node negative ILCs only). The reproducibility of the solid and alveolar ILCs on the one hand and grade 3 ductal cancers on the other therefore becomes important. Alveolar cancers are easy to recognize and should not offer a serious problem if the pathologist is well trained. We have used strict, widely available and applicable WHO 2003 criteria to guarantee correct classification which, in our hands as experts in breast pathology was well reproducible, though in a routine practice of general surgical pathologists, reproducibility may sometimes be less good. This can be regarded as a drawback to give histologic subtype in ILCs such a prominent prognostic role. However, this objection is much less important than it seems as immunohistochemistry (E-Cadherin, oestrogen receptor) is of great help. Even if pathologists erroneously classify a lobular cancer as ductal, then MAI can still predict prognosis correctly if MAI is $\geq 10$. Only in the relatively few cases with MAI 6-10 (which occurs in only $10 \%$ of all ILCs), one would get a too favorable prognostic impression if the cancer with a MAI between 5 and 10 is erroneously classified as a ductal grade 3, instead of a solid ILC. Strict use of the classification criteria, and additional immunohistochemical E-cadherin, and ER analysis is therefore of importance and enhances the significance of an accurate pathologic diagnosis in determining the true outcome of the patient.

We conclude that in node negative lobular invasive cancers, histologic subtype and MAI with a threshold of five (rather than MAI with a threshold of ten, tubular formation, nuclear atypia and grade) have independent and prognostic value.

Acknowledgments The MMMCP study is supported by grant \#281398 of the Zorg Onderzoek Nederland (ZON) and grants \#06-212 and $07-218$ of the SBDM. Besides the authors, the project has been made possible by the continuing collaboration of all the specialists, bioengineers and administrative collaborators of the MMMCP project.

\section{References}

1. Ellis IO, Schnitt SJ, Sastre-Garau X (2003) Invasive breast carcinoma. In: Tavassoli FA, Devilee $P$ et al (eds) Pathology and genetics of tumours of the breast and female genital organs: WHO classification of tumours series. IARC press, Lyon, pp 13-48

2. Mathieu MC, Rouzier R, Llombart-Cussac A et al (2004) The poor responsiveness of infiltrating lobular breast carcinomas to neoadjuvant chemotherapy can be explained by their biological profile. Eur J Cancer 40:342-351. doi:10.1016/j.ejca.2003.08.015

3. Cocquyt V, Van Belle S (2005) Lobular carcinoma in situ and invasive lobular cancer of the breast. Curr Opin Obstet Gynecol 17:55-60. doi:10.1097/00001703-200502000-00010

4. Dixon JM, Anderson TJ, Page DL et al (1982) Infiltrating lobular carcinoma of the breast. Histopathology 6:149-161. doi:10.1111/ j.1365-2559.1982.tb02712.x 
5. Arpino G, Bardou VJ, Clark GM, Elledge RM (2004) Infiltrating lobular carcinoma of the breast: tumor characteristics and clinical outcome. Breast Cancer Res 6:R149-R156. doi:10.1186/bcr767

6. Reis-Filho JS, Simpson PT, Jones C, Steele D, Mackay A, Iravani $\mathrm{M}$ et al (2005) Pleomorphic lobular carcinoma of the breast: role of comprehensive molecular pathology in characterization of an entity. J Pathol 207:1-13. doi:10.1002/path.1806

7. Baak JP, van Diest PJ, Voorhorst FJ, van der Wall E, Beex LV, Vermorken JB et al (2005) Prospective multicenter validation of the independent prognostic value of the mitotic activity index in lymph node-negative breast cancer patients younger than 55 years. J Clin Oncol 23(25):5993-6001. doi:10.1200/JCO.2005.05.511

8. Baak JP, van Diest PJ, Janssen EA et al (2008) Proliferation accurately identifies the high-risk patients among small, lowgrade, lymph node-negative invasive breast cancers. Ann Oncol 19(4):649-654. doi:10.1093/annonc/mdm535

9. McShane LM, Altman DG, Sauerbrei W et al (2005) Reporting recommendations for tumor marker studies. J Clin Oncol 23(36):9067-9072

10. Baak JPA, Snijders W, van Diermen B et al (2003) Prospective multicenter validation confirms the prognostic superiority of the
ECPI in FIGO-1 endometrial carcinoma. J Clin Oncol 23:42144221. doi:10.1200/JCO.2003.02.087

11. Jannink I, Bennen JN, Blaauw J et al (1995) At convenience and systematic random sampling: effects on the prognostic value of nuclear area assessments in breast cancer patients. Breast Cancer Res Treat 36:55-60. doi:10.1007/BF00690185

12. Fleege JC, van Diest PJ, Baak JPA (1993) Systematic random sampling for selective interactive nuclear morphometry in breast cancer sections. Refinement and multiobserver evaluation. Anal Quant Cytol Histol 15:281-289

13. Skaland I, van Diest PJ, Janssen EAM et al (2008) Prognostic differences of WHO-assessed mitotic activity index (MAI) and mitotic impression by quick scanning in invasive ductal breast cancer patients under 55 years of age. Hum Pathol 39(4):584590. doi:10.1016/j.humpath.2007.08.016

14. Rakha EA, El-Sayed ME, Menon S et al (2008) Histologic grading is an independent prognostic factor in invasive lobular carcinoma of the breast. Breast Cancer Res Treat 111(1):121-127 (Epub 2007 Oct 11) 